\title{
Portfolio selection under distributional uncertainty: A relative robust CVaR approach
}

\section{$\operatorname{AUTHOR}(\mathrm{S})$ :}

Huang, Dashan; Zhu, Shushang; Fabozzi, Frank J.; Fukushima, Masao

\section{CITATION:}

Huang, Dashan ... [et al]. Portfolio selection under distributional uncertainty: A relative robust CVaR approach. European Journal of Operational Research 2010, 203(1): 185-194

\author{
ISSUE DATE: \\ 2010-05 \\ URL: \\ http://hdl.handle.net/2433/87374 \\ RIGHT: \\ c 2009 Elsevier B.V. All rights reserved.
}




\title{
Portfolio Selection under Distributional Uncertainty: A Relative Robust CVaR Approach*
}

\author{
Dashan Huang $^{\dagger}$ Shushang Zhu ${ }^{\ddagger}$ Frank J. Fabozzi ${ }^{\S}$ Masao Fukushima
}

First version: March 11, 2007

This version: February 5, 2009

\begin{abstract}
Robust optimization, one of the most popular topics in the field of optimization and control since the late 1990s, deals with an optimization problem involving uncertain parameters. In this paper, we consider the relative robust conditional value-at-risk portfolio selection problem where the underlying probability distribution of portfolio return is only known to belong to a certain set. Our approach not only takes into account the worstcase scenarios of the uncertain distribution, but also pays attention to the best possible decision with respect to each realization of the distribution. We also illustrate how to construct a robust portfolio with multiple experts (priors) by solving a sequence of linear programs or a second-order cone program.
\end{abstract}

Keywords: Conditional value-at-risk; worst-case conditional value-at-risk; relative robust conditional value-at-risk; portfolio selection problem, linear programming.

Subject Classification: O.R. Applications

\footnotetext{
${ }^{*}$ We would like to thank two anonymous referees for constructive comments and suggestions that have clearly aided us in improving the paper. This work was partially supported by the National Science Foundation of China (No. 70401009) (S.S. Zhu) and the Scientific Research Grant-in-Aid from Japan Society for the Promotion of Science (M. Fukushima).

${ }^{\dagger}$ Olin School of Business, Washington University, St. Louis, MO 63130, USA. Email: huangdas@wustl.edu.

${ }^{\ddagger}$ Department of Management Science, School of Management, Fudan University, Shanghai, 200433, China. Email: sszhu@fudan.edu.cn.

${ }^{\S}$ School of Management, Yale University, New Haven, CT06520, USA. Email: frank.fabozzi@yale.edu.

${ }^{\top}$ Corresponding author. Department of Applied Mathematics and Physics, Graduate School of Informatics, Kyoto University, Kyoto, 606-8501, Japan. Tel.: +81 75753 5519; Fax: +81 75753 4756. Email: fuku@amp.i.kyoto-u.ac.jp.
} 


\section{Introduction}

Based on mean-variance analysis, Markowitz (1952) suggests that investors should decide the allocation of their investment in two stages: 1) estimate the input parameters (a belief about the future performance of available securities) from empirical observations or subjective experience and 2) select the portfolio on the basis of a trade-off between risk and return. In practice, however, a plethora of empirical studies (see, for example, Best and Grauer, 1991; Broadie, 1992; Chopra, 1993; Chopra and Ziemba, 1993) show that a small perturbation of the inputs may lead to a large change in the optimal portfolio, implying that the parameters should be estimated precisely enough. However, the input parameters are estimated from real data, which are often prone to errors. Thus, the lack of robustness of inputs usually entails extreme positions in the assets of the optimal portfolio and delivers a poor out-of-sample performance (Black and Litterman, 1992; Litterman, 2003).

There are two standard methods extensively adopted in the literature to deal with estimation errors: the Bayesian approach and the robust optimization approach. However, the Bayesian approach is neutral to uncertainty in the sense of Knight (1921) because it assumes a single prior on the portfolio distribution (Garlappi et al., 2007). In contrast, the robust optimization approach, due to multiple priors, has obtained prodigious success since the late 1990s, especially in the field of optimization and control with uncertain parameters (Ben-Tal and Nemirovski, 1998, 1999; El Ghaoui and Lebret, 1997; Goldfarb and Iyengar, 2003a; Stinstra and Hertog, 2008). With respect to portfolio selection, the major contributions have come in the 21st century (see, for example, Costa and Paiva, 2002; Goldfarb and Iyendar, 2003b; El Glaoui et al., 2003; Tütüncü and Koenig, 2004; Zhu and Fukushima, 2005; Lu, 2006; Huang et al., 2007, 2008; Gülpinar and Rustem, 2007; Liesiö et al., 2007; Zhu et al., 2007; Shen and Zhang, 2008; Natarajan et al., 2008). For a complete discussion of robust portfolio optimization and the associated solution methods, see Fabozzi et al. (2007, 2009) and the references therein.

All the above mentioned literature about robust optimization can be regarded as absolute robust optimization, which assumes that the parameters follow a uniform distribution in the uncertain set, and hence focus exclusively on the extreme worst-case situation of the uncertain parameters. However, if the extreme worst-case scenario in the uncertainty set takes place disproportionally (not uniformly, and they are not very likely to occur), then the final optimal portfolio will be very conservative because it only depends on the extreme small probability for the parameters. Yet, how to measure the performance/cost of robustness is an important and complicated problem in practice, since the success of robustness is at the cost of the portfolio return/reward. The more robustness, that is, the larger magnitude the uncertain set, the lower the worst-case expected return.

In this paper, we propose a relative robust approach to deal with the portfolio selection 
problem in the relative robust conditional value-at-risk (RCVaR) framework. Compared with Zhu and Fukushima (2005) where the main focus is on the worst-case scenarios of the parameters that may rarely be realized in practice, ${ }^{1}$ we also consider the most tractable scenarios which may result in the most optimistic portfolio choices. We realize this strategy by measuring the portfolio risk relative to a benchmark risk, which is the minimum risk if the parameters represent the investor's belief precisely and do not suffer from any estimation error with given data. Although it is not possible to acknowledge the parameters through statistical estimation, we may believe with certainty that they belong to a certain set associated with the estimated values. Thus, we want to construct a portfolio so that the relative risk is as small as possible with respect to each scenario of the uncertain parameters in this set. The advantages of our relative risk approach can be summarized as: 1) the optimal portfolio resulting from this approach is less conservative than that arising from the classical absolute robust approach, and 2) we can formulate the portfolio selection problem as a set of linear programs which can be efficiently solved.

The paper is organized as follows. In Section 2, we introduce the concept of RCVaR, followed by an investigation of the minimization of this risk measure in the case where the underlying probability distribution of the portfolio return belongs to a certain set in Section 3. In Section 4, we apply our model to robust portfolio optimization with multiple experts, illustrated by equity portfolio management and hedge fund management. A summary of our findings is provided in Section 5.

\section{Relative Robust Conditional Value-at-Risk}

Suppose there exist $n$ risky assets for an investor to construct portfolios. Let the random vector $y=\left(y_{1}, \cdots, y_{n}\right)^{\top} \in \Re^{n}$ denote uncertain returns of the $n$ risky assets, and $x=$ $\left(x_{1}, \cdots, x_{n}\right)^{\top} \in X \subseteq \Re^{n}$ denote the amount of the portfolio to be invested in the $n$ risky assets, where ${ }^{\top}$ means transposition. Then the portfolio return is defined as $x^{\top} y$, and the loss function is the negative of the return, i.e.,

$$
f(x, y)=-x^{\top} y
$$

For fixed $x \in X$ and known probability distribution $\pi(y)$, the probability of the loss not exceeding a threshold $\zeta$ is given by

$$
\Psi(x, \zeta)=\int_{f(x, y) \leq \zeta} \pi(y) d y .
$$

\footnotetext{
${ }^{1}$ The parameter uncertainty in the conditional value-at-risk (CVaR) framework corresponds to the distribution uncertainty. In this sense, the method in Zhu and Fukushima (2005) is in the spirit of Krokhma et al., (2003, 2004). Natarajan et al. (2005) also discussed the worst-case CVaR under moment cone uncertainty.
} 
Given a confidence level $\alpha$, the value-at-risk (VaR) associated with the portfolio $x$ is defined as

$$
\operatorname{VaR}_{\alpha}(x, \pi)=\min \{\zeta \in \Re: \Psi(x, \zeta) \geq \alpha\} .
$$

The corresponding conditional value-at-risk (CVaR) is defined by Rockafellar and Uryasev (2000) as the conditional expectation of the loss of the portfolio exceeding or equal to VaR, i.e.,

$$
\operatorname{CVaR}_{\alpha}(x, \pi)=\frac{1}{1-\alpha} \int_{f(x, y) \geq \operatorname{VaR}_{\alpha}(x, \pi)} f(x, y) \pi(y) d y .
$$

Moreover, Rockafellar and Uryasev $(2000,2002)$ prove that CVaR has an equivalent definition as

$$
\operatorname{CVaR}_{\alpha}(x, \pi)=\min _{\zeta \in \Re} F_{\alpha}(x, \zeta, \pi)
$$

where

$$
F_{\alpha}(x, \zeta, \pi)=\zeta+\frac{1}{1-\alpha} \mathrm{E}_{\pi}[f(x, y)-\zeta]^{+},
$$

where $\mathrm{E}_{\pi}(\cdot)$ denotes the expectation with respect to $\pi$ and $[t]^{+}=\max \{t, 0\}$. More specifically, if the portfolio returns are multivariate normally distributed $(y \sim \mathcal{N}(\bar{y}, \Sigma))$, CVaR reduces to

$$
\operatorname{CVaR}_{\alpha}(x, \pi)=k_{\alpha}\left\|\Sigma^{\frac{1}{2}} x\right\|-x^{\top} \bar{y}
$$

where

$$
k_{\alpha}=\frac{-\int_{-\infty}^{\Phi^{-1}(1-\alpha)} t \phi(t) d t}{1-\alpha}
$$

and $\|\cdot\|$ represents the standard Euclidean norm, $\phi(\cdot)$ and $\Phi(\cdot)$ are the standard normal density and cumulative distribution functions, respectively. On the other hand, if the distribution is not normal, to deal with the calculation of the multivariate integral, Rockafellar and Uryasev (2000, 2002) show that discretization sampling via Monte Carlo simulation can be regarded as a good approximation, which will make the problem a standard linear program. This means that we may assume that $y \in \Re^{n}$ follows a discrete distribution $\left\{y_{[1]}, y_{[2]}, \cdots, y_{[S]}\right\}$ with probabilities $\operatorname{Pr}\left\{y_{[s]}\right\}=\pi_{s}$ such that $\sum_{s=1}^{S} \pi_{s}=1, \pi_{s} \geq 0, s=1, \cdots, S$, and therefore

$$
F_{\alpha}(x, \zeta, \pi)=\zeta+\frac{1}{1-\alpha} \sum_{s=1}^{S} \pi_{s}\left[f\left(x, y_{[s]}\right)-\zeta\right]^{+},
$$

where $S$ is the number of support points of $\pi$.

In general, minimizing $\mathrm{CVaR}$ over $x \in X$ is equivalent to minimizing $F_{\alpha}(x, \zeta, \pi)$ over $(x, \zeta) \in X \times \Re$, i.e.

$$
\min _{x \in X} \operatorname{CVaR}_{\alpha}(x, \pi)=\min _{(x, \zeta) \in X \times \Re} F_{\alpha}(x, \zeta, \pi),
$$


which implies that a pair $\left(x^{*}, \zeta^{*}\right)$ solves $\min _{(x, \zeta) \in X \times \Re} F_{\alpha}(x, \zeta, \pi)$ if and only if $x^{*}$ minimizes $\mathrm{CVaR}_{\alpha}(x, \pi)$ over $X$. Moreover, $F_{\alpha}(x, \zeta, \pi)$ is convex with respect to $(x, \zeta)$ and $\operatorname{CVaR}_{\alpha}(x, \pi)$ is convex with respect to $x$, and hence the above problem can be efficiently solved by an interior-point algorithm (Rockafellar and Uryasev; 2000, 2002).

Instead of assuming the precise distribution of the random vector $y$, Zhu and Fukushima (2005) consider the case where the probability distribution $\pi$ is only known to belong to a certain set $\mathscr{P}$ of distributions, and define the worst-case CVaR (WCVaR) with respect to $\mathscr{P}$ $\mathrm{as}^{2}$

$$
\operatorname{WCVaR}_{\alpha}(x)=\sup _{\pi \in \mathscr{P}} \operatorname{CVaR}_{\alpha}(x, \pi)
$$

It should be mentioned that there are different definitions of robust optimization in the literature, which can be generally classified into two categories - absolute robust optimization and relative robust optimization. Absolute robust optimization, also called worst-case optimization, assumes that the final decision is only influenced by the worst case of an uncertain input parameter set (worst-case CVaR in the sense of Zhu and Fukushima (2005) belongs to this category). In contrast, relative robust optimization considers the uncertain parametric optimization problem in a relative sense, which pays attention not only to the worst-case decision but also to the best possible decision for each realization of the input parameters. Specifically, the relative robust CVaR (RCVaR) for fixed $x \in X$ with respect to $\mathscr{P}$ can be defined as

$$
\operatorname{RCVaR}_{\alpha}(x)=\sup _{\pi \in \mathscr{P}}\left\{\operatorname{CVaR}_{\alpha}(x, \pi)-\operatorname{CVaR}_{\alpha}\left(z^{*}(\pi), \pi\right)\right\}
$$

where $z^{*}(\pi)=\operatorname{argmin}_{z \in X} \operatorname{CVaR}_{\alpha}(z, \pi) .^{3}$

We can explain the concept of RCVaR in the following way. Suppose $X$ is the feasible set of portfolio and $\pi$ is the true distribution of the random vector $y$. Then, $\operatorname{CVaR}_{\alpha}\left(z^{*}(\pi), \pi\right)$ denotes the minimum CVaR of the portfolio in $X$ with respect to the distribution $\pi$. Thus, $\operatorname{CVaR}_{\alpha}(x, \pi)-\operatorname{CVaR}_{\alpha}\left(z^{*}(\pi), \pi\right)$ represents the risk of the portfolio $x$ relative to the benchmark portfolio $z^{*}(\pi)$ corresponding to $\pi$. However, because it is not possible to acknowledge with certainty the distribution $\pi$ precisely, we can only construct a confidence region $\mathscr{P}$ for $\pi$ from the experimental data or subjective experience, and try to make $\operatorname{CVaR}_{\alpha}(x, \pi)-$ $\operatorname{CVaR}_{\alpha}\left(z^{*}(\pi), \pi\right)$ as small as possible for all possible $\pi \in \mathscr{P}$ when we choose portfolio $x$ in the feasible set $X$. Thus, $\sup _{\pi \in \mathscr{P}}\left\{\operatorname{CVaR}_{\alpha}(x, \pi)-\mathrm{CVaR}_{\alpha}\left(z^{*}(\pi), \pi\right)\right\}$ is the potential greatest

\footnotetext{
${ }^{2}$ Robust/worst-case optimization is only a concept or a strategy, which has different meanings in the literature. Although some researchers look at CVaR as a robust optimization concept in the sense that $\min _{x \in X} \operatorname{CVaR}(x, \pi)$ can be formulated as a min-max optimization problem, see, for example, Rockafellar et al. (2006), we discuss robust optimization in the context of $\min _{x \in X} \max _{\pi \in \mathscr{P}} \operatorname{CVaR}(x, \pi)$.

${ }^{3}$ Although WCVaR is a coherent risk measure (Zhu and Fukushima, 2005), RCVaR is not, due to the term of $\mathrm{CVaR}_{\alpha}\left(z^{*}(\pi), \pi\right)$ that makes RCVaR neither subadditive nor positive homogenous.
} 
relative risk incurred by $x$ with respect to $\mathscr{P} .{ }^{4}$

Up to now, RCVaR is just a concept because it is not ready for application. In the following, we will elaborate on RCVaR with respect to some special structures of the uncertain distribution set $\mathscr{P}$, and at the same time, make the formulations as tractable as possible.

\section{Problem Formulation}

Suppose there exist finite multiple rival forecasts to represent the distribution of $y$, i.e.,

$$
\pi \in \mathscr{P}_{\mathrm{M}}=\left\{\pi^{i}: i=1, \cdots, l\right\},
$$

where $\pi^{i}$ is the $i$-th possible distribution and $l$ is the number of possible distributions. In this case, the investor may obtain estimation (prior) of $\pi$ from $l$ approaches and does not have any judgement of which estimation (prior) is the best, and hence, the investor tends to incorporate all existing rival scenarios into the investment decision simultaneously.

Hence, we can rewrite $\mathrm{RCVaR}$ with respect to $\mathscr{P}_{\mathrm{M}}$ as

$$
\begin{aligned}
\operatorname{RCVaR}_{\alpha}(x) & =\max _{\pi \in \mathscr{P}_{\mathrm{M}}}\left\{\operatorname{CVaR}_{\alpha}(x, \pi)-\operatorname{CVaR}_{\alpha}\left(z^{*}(\pi), \pi\right)\right\} \\
& =\max _{\pi \in \mathscr{P}_{\mathrm{M}}}\left\{\min _{\zeta \in \Re} F_{\alpha}(x, \zeta, \pi)-\min _{(\boldsymbol{z}, \eta) \in X \times \Re} F_{\alpha}(z, \eta, \pi)\right\}, \\
& =\max _{i \in \mathcal{I}}\left\{\min _{\zeta \in \Re} F_{\alpha}\left(x, \zeta, \pi^{i}\right)-\gamma^{*}\left(\pi^{i}\right)\right\},
\end{aligned}
$$

where $\mathcal{I}=\{1,2, \cdots, l\}$ and

$$
\gamma^{*}\left(\pi^{i}\right)=\min _{(z, \zeta) \in X \times \Re} F_{\alpha}\left(z, \zeta, \pi^{i}\right)
$$

So that the robust portfolio selection problem with RCVaR risk measure becomes

$$
\min _{x \in X} \max _{i \in \mathcal{I}}\left\{\min _{\zeta \in \Re} F_{\alpha}\left(x, \zeta, \pi^{i}\right)-\gamma^{*}\left(\pi^{i}\right)\right\}
$$

\footnotetext{
${ }^{4}$ Alternatively, we can also define the RCVaR as the best worst-case percentage regret from optimality, i.e.,

$$
\operatorname{RCVaR}_{\alpha}(x)=\sup _{\pi \in \mathscr{P}} \frac{\operatorname{CVaR}_{\alpha}(x, \pi)-\operatorname{CVaR}_{\alpha}\left(z^{*}(\pi), \pi\right)}{\operatorname{CVaR}_{\alpha}\left(z^{*}(\pi), \pi\right)} .
$$

In this definition, it is tacitly assumed that $\operatorname{CVaR}_{\alpha}\left(z^{*}(\pi), \pi\right)>0$. Actually, this requirement can be easily

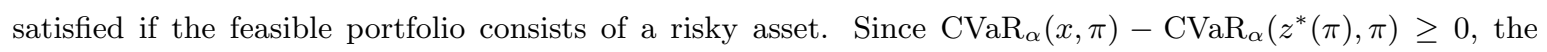
$\operatorname{RCVaR}_{\alpha}(x)$ is nonnegative for any $x \in X$. Therefore, the minimization of RCVaR over $x \in X$ with respect to $\pi \in \mathscr{P}$ can be formulated as the following optimization problem:
}

$$
\min _{(x, \theta) \in X \times \Re}\left\{\theta: \operatorname{CVaR}_{\alpha}(x, \pi)-(1+\theta) \operatorname{CVaR}_{\alpha}\left(z^{*}(\pi), \pi\right) \leq 0, \forall \pi \in \mathscr{P}\right\} .
$$


this can be equivalently formulated as the following problem:

$$
\begin{array}{cl}
\min _{(x, \theta) \in X \times \Re} & \theta \\
\text { s.t. } & \min _{\zeta \in \Re} F_{\alpha}\left(x, \zeta, \pi^{i}\right)-\gamma^{*}\left(\pi^{i}\right) \leq \theta, \quad i=1, \cdots, l .
\end{array}
$$

Now let us consider the following auxiliary problem:

$$
\begin{array}{ll}
\min _{(x, \zeta, \theta) \in X \times \Re^{l} \times \Re} & \theta \\
\text { s.t. } & F_{\alpha}\left(x, \zeta_{i}, \pi^{i}\right)-\gamma^{*}\left(\pi^{i}\right) \leq \theta, \quad i=1, \cdots, l .
\end{array}
$$

The following theorem shows that problem (7) and problem (8) are equivalent.

Theorem 1 Suppose that $\left(x^{*}, \theta^{*}\right)$ solves problem (7), then $\left(x^{*}, \zeta^{*}, \theta^{*}\right)$ solves problem (8) with $\zeta_{i}^{*}=\operatorname{argmin}_{\zeta_{i} \in \Re} F_{\alpha}\left(x^{*}, \zeta_{i}, \pi^{i}\right)$ for $i \in \mathcal{I}$; Conversely, suppose that $(\bar{x}, \bar{\zeta}, \bar{\theta})$ solves problem (8), then $(\bar{x}, \bar{\theta})$ solves problem (7).

Proof. Suppose that $\left(x^{*}, \theta^{*}\right)$ solves $(7)$. It is obvious that $\left(x^{*}, \zeta^{*}, \theta^{*}\right)$ is a feasible solution to (8), where $\zeta_{i}^{*}=\operatorname{argmin}_{\zeta_{i} \in \Re} F_{\alpha}\left(x^{*}, \zeta_{i}, \pi^{i}\right)$. If $\left(x^{*}, \zeta^{*}, \theta^{*}\right)$ does not solve (8), then there exists a triple $(\bar{x}, \bar{\zeta}, \bar{\theta})$ such that it is feasible to $(8)$ and $\bar{\theta}<\theta^{*}$. Notice that for any $i \in \mathcal{I}$,

$$
\min _{\zeta \in \Re} F_{\alpha}\left(\bar{x}, \zeta, \pi^{i}\right)-\gamma^{*}\left(\pi^{i}\right) \leq F_{\alpha}\left(\bar{x}, \bar{\zeta}_{i}, \pi^{i}\right)-\gamma^{*}\left(\pi^{i}\right) \leq \bar{\theta}
$$

Thus $(\bar{x}, \bar{\theta})$ is feasible to $(7)$. This contradicts the hypothesis that $\left(x^{*}, \theta^{*}\right)$ is an optimal solution to $(7)$, since $\bar{\theta}<\theta^{*}$. Therefore, $\left(x^{*}, \zeta^{*}, \theta^{*}\right)$ must solve (8).

Conversely, suppose $(\bar{x}, \bar{\zeta}, \bar{\theta})$ solves (8). By (9), it is obvious that $(\bar{x}, \bar{\theta})$ is feasible to (7). If $(\bar{x}, \bar{\theta})$ does not solve $(7)$, then there exists a pair $\left(x^{*}, \theta^{*}\right)$ that solves $(7)$ such that $\theta^{*}<\bar{\theta}$. By the first part of the proof we have that $\left(x^{*}, \zeta^{*}, \theta^{*}\right)$ solves (8) with $\zeta_{i}^{*}=$ $\operatorname{argmin}_{\zeta_{i} \in \Re} F_{\alpha}\left(x^{*}, \zeta_{i}, \pi^{i}\right)$, which contradicts the hypothesis that $(\bar{x}, \bar{\zeta}, \bar{\theta})$ is an optimal solution to $(8)$, since $\theta^{*}<\bar{\theta}$. Therefore, $(\bar{x}, \bar{\theta})$ must solve $(7)$. This completes the proof.

To make problem (8) more tractable, we use an artificial variable $v=\left(v^{1} ; \cdots ; v^{l}\right)$ and rewrite it as

$$
\begin{array}{cl}
\min _{(x, \zeta, \theta, \boldsymbol{v}) \in X \times \Re^{l} \times \Re \times \Re^{m}} & \theta \\
\text { s.t. } & \zeta_{i}+\frac{1}{1-\alpha}\left(\pi^{i}\right)^{\top} v^{i}-\gamma^{*}\left(\pi^{i}\right) \leq \theta, \\
& v_{s}^{i} \geq f\left(x, y_{[s]}^{i}\right)-\zeta_{i}, \\
& v_{s}^{i} \geq 0, s=1, \cdots, S, i=1, \cdots, l,
\end{array}
$$

where $v^{i}=\left(v_{1}^{i}, \cdots, v_{s}^{i}\right)^{\top}, \pi^{i}=\left(\pi_{1}^{i}, \cdots, \pi_{S}^{i}\right)^{\top}$, and $m=l S$. From (3), it is not difficult to see that this problem is equivalent to $(8)$. 
Usually, the feasible portfolio set is specified as

$$
X=\left\{x: e^{\top} x=1, \underline{x} \leq x \leq \bar{x}, \min _{\pi \in \mathscr{P}_{\mathrm{M}}} \mathrm{E}_{\pi}\left(x^{\top} y\right) \geq \mu\right\}
$$

where $e=(1, \cdots, 1)^{\top} \in \Re^{n}, e^{\top} x=1$ is the budget constraint, the inequalities $\underline{x} \leq x \leq \bar{x}$ ensure diversification of an optimal portfolio with given lower and upper bounds $\underline{x}$ and $\bar{x}$, and the target constraint $\min _{\pi \in \mathscr{P}_{\mathrm{M}}} \mathrm{E}_{\pi}\left(x^{\top} y\right) \geq \mu$ indicates that the worst-case expected return the investor can obtain should be at least $\mu$. Specifically, in the case of finite rival-scenario uncertainty, the target constraint is equivalent to

$$
x^{\top} \bar{y}^{i} \geq \mu, \quad i=1, \cdots, l,
$$

where $\bar{y}^{i}$ represents the expected value of $y$ with respect to the $i$-th likelihood distribution $\pi^{i}$. Additionally, the feasible portfolio set in problem (5) for each $i$ reduces to

$$
X_{i}=\left\{z: e^{\top} z=1, \underline{x} \leq z \leq \bar{x}, z^{\top} \bar{y}^{i} \geq \mu\right\},
$$

which also applies to the case of infinite rival-scenario uncertainty later.

In practice, one may also impose additional constraints on $x$ to obtain a more reasonable portfolio, such as the transaction cost constraint or the net weight-adjustment constraint as in Fabozzi et al. (2007).

In summary, the minimization of $\operatorname{RCVaR}_{\alpha}(x)$ over $X$ with finite rival-scenario uncertainty can be formulated as the following optimization problem:

$\left(P_{1}\right)$

$$
\begin{array}{cl}
\min _{(x, \zeta, \theta, v) \in \Re^{n} \times \Re^{l} \times \Re \times \Re^{m}} & \theta \\
\text { s.t. } & e^{\top} x=1, \\
& \underline{x} \leq x \leq \bar{x}, \\
& x^{\top} \bar{y}^{i} \geq \mu, \\
& \zeta_{i}+\frac{1}{1-\alpha}\left(\pi^{i}\right)^{\top} v^{i}-\gamma^{*}\left(\pi^{i}\right) \leq \theta, \\
& v_{s}^{i} \geq-x^{\top} y_{[s]}^{i}-\zeta_{i}, \\
& v_{s}^{i} \geq 0, s=1, \cdots, S, i=1, \cdots, l,
\end{array}
$$

which requires to solve the programs (5) first for each $i=1, \cdots, l$. Fortunately, all these problems are linear programs and hence can be solved efficiently by the well-known interiorpoint methods or simplex method. More specifically, if all the rival scenarios have the common support space, i.e., $y_{[s]}^{i}=y_{[s]}^{j}, \forall i \neq j$, then we have $v^{i}=v^{j}$, implying that $\left(P_{1}\right)$ has only $n+1+l+S$ variables, far less than $n+1+l+l S$.

It should be mentioned that if $\gamma^{*}\left(\pi^{i}\right)=0$ in $\left(P_{1}\right)$, the problem reduces to the worst-case CVaR portfolio optimization problem, and more particularly, if $l=1,\left(P_{1}\right)$ reduces to the nominal CVaR portfolio selection problem. 


\subsection{Normal distribution case}

As noted above, in the general case, formulation $\left(P_{1}\right)$ needs a large number of samples. However, this may not be so convenient in some applications. In this subsection, we address the problem of RCVaR in the special case where the portfolio return has a multivariate normal distribution but the moments of the return are only known to belong to a given set, that is

$$
\pi \in \mathscr{P}_{(\bar{y}, \Sigma)}
$$

For this case, we can simply formulate the problem as a second-order cone program.

Suppose that the feasible portfolio set is given by (10). Then, the RCVaR portfolio selection problem becomes

$$
\begin{aligned}
& \min _{x \in X} \max _{\pi \in \mathscr{P}_{(\bar{y}, \Sigma)}}\left\{\operatorname{CVaR}_{\alpha}(x, \pi)-\operatorname{CVaR}_{\alpha}\left(z^{*}(\pi), \pi\right)\right\} \\
= & \min _{x \in X} \max _{\pi \in \mathscr{P}_{(\bar{y}, \Sigma)}}\left\{k_{\alpha}\left\|\Sigma^{\frac{1}{2}} x\right\|-x^{\top} \bar{y}-\min _{z \in X}\left\{k_{\alpha}\left\|\Sigma^{\frac{1}{2}} z\right\|-z^{\top} \bar{y}\right\}\right\},
\end{aligned}
$$

where $k_{\alpha}$ is defined as in (2). Particularly, according to Rockafellar and Uryasev (2000), if $\alpha>0.5, \underline{x}=-\infty$ and $\bar{x}=+\infty$, problem (11) can be written as

$$
\min _{x \in X} \max _{\pi \in \mathscr{P}(\bar{y}, \Sigma)}\left\{k_{\alpha}\left\|\Sigma^{\frac{1}{2}} x\right\|-x^{\top} \bar{y}-k_{\alpha}\left\|\Sigma^{-\frac{1}{2}} d\right\|-\mu\right\}
$$

where

$$
d=\frac{\bar{y}(c \mu-b)+e(a-b \mu)}{a c-b^{2}},
$$

$a=\bar{y}^{\top} \Sigma^{-1} \bar{y}, b=\bar{y}^{\top} \Sigma^{-1} e$, and $c=e^{\top} \Sigma^{-1} e$.

Suppose $(\bar{y}, \Sigma)$ is only known to belong to a finite rival-scenario uncertainty set, i.e.,

$$
\pi \in \mathscr{P}_{(\bar{y}, \Sigma)}^{M}=\left\{\left(\bar{y}^{i}, \Sigma^{i}\right), i=1, \cdots, l\right\} .
$$

Then, together with Theorem 1, problem (11) reduces to

$$
\min _{(x, \theta) \in X \times \Re}\left\{\theta: k_{\alpha}\left\|\left(\Sigma^{i}\right)^{\frac{1}{2}} x\right\|-x^{\top} \bar{y}^{i}-\gamma^{*}\left(\bar{y}^{i}, \Sigma^{i}\right) \leq \theta, i=1, \cdots, l\right\},
$$

where $\gamma^{*}\left(\bar{y}^{i}, \Sigma^{i}\right)=\min _{z \in X}\left\{k_{\alpha}\left\|\left(\Sigma^{i}\right)^{\frac{1}{2}} z\right\|-z^{\top} \bar{y}^{i}\right\}$. Similarly to the finite rival-scenario uncertainty in the previous section, the feasible portfolio set $X$ can be specified as (10). Therefore, RCVaR portfolio selection with finite rival-moment uncertainty can be explicitly formulated as the following minimization problem over $(x, \theta) \in \Re^{n} \times \Re$ :

$$
\begin{aligned}
\left(P_{2}\right) \quad \min \quad \theta & \\
\text { s.t. } \quad & e^{\top} x=1, \\
& \underline{x} \leq x \leq \bar{x}, \\
& x^{\top} \bar{y}^{i} \geq \mu, \\
& k_{\alpha}\left\|\left(\Sigma^{i}\right)^{\frac{1}{2}} x\right\|-x^{\top} \bar{y}^{i}-\gamma^{*}\left(\bar{y}^{i}, \Sigma^{i}\right) \leq \theta, i=1, \cdots, l .
\end{aligned}
$$


Clearly, $\left(P_{2}\right)$ is a second-order cone program that can be solved efficiently by interior-point methods (see, for example, Ben-Tal and Nemirovski, 1998).

It should be mentioned that the estimation of the mean and covariance has received considerable attention in both theory and practice (see Lutgens and Schotman (2008), Schöttle and Werner (2006), Garlappi et al. (2007), and references therein for details).

\subsection{Specification of uncertainty set}

While there is a large body of literature concerned with the formulation of robust portfolio selection, the discussion exclusively on how to specify the size and shape of the set of the uncertain parameter in an attractive manner is limited; the size of the set ensures the probability that the uncertain parameter takes on value in the set, while the shape of the set determines the complexity of the robust optimization problem.

In practice, a distribution is often completely determined by its parameters. In this sense, we may write $\pi^{i}$ as $\pi\left(\Theta_{i}\right)$. To identify $\Theta_{i}$, two alternative approaches have been extensively employed in both theory and practice. One is empirical observation: collecting a set of behaved historical data to estimate the parameters of the likelihood distribution. For example, if we consider the returns following a joint normal distribution, the corresponding mean and covariance of the portfolio returns can be estimated immediately from historical returns. However, due to estimate errors, we can only obtain a confidence region $\Theta$ of the parameters, and we believe with certainty that the most possible parameters $\Theta_{i}$ belong to $\Theta$, i.e., $\Theta_{i} \in \Theta$. Thus, we may choose proper $\Theta_{i}$ in $\Theta$ to construct the likelihood distribution $\pi\left(\Theta_{i}\right)$.

The other approach is expert prediction: all the different distributions predicted by a group of experts can be regarded as the likelihood distributions (Lutgens and Schotman, 2006). Actually, the success of quantitative approaches to portfolio selection relies on the return model employed. While each alternative return model supplies the portfolio manager with a specific set of parameters such as the mean and covariance of the returns, there is no consensus among the experts on which return model is most appropriate, and hence they will recommend different parameters for the portfolio return distribution. Apart from relying on one particular expert, we may combine the information from a number of experts and consider each of their predictions as a likelihood distribution $\pi\left(\Theta_{i}\right) .{ }^{5}$

\footnotetext{
${ }^{5}$ We mention here that how to determine the number of experts or what kinds of experts should be hired are two important issues in practical application, and unfortunately, to our knowledge, there is no consensus on these specifications, including Lutgens and Schotman (2008) and Garlappi et al. (2007). However, our paper is mainly concerned with the integration of multiple beliefs (predictions) to select a relative robust portfolio.
} 


\section{Application to Robust Portfolio Management with Multiple Experts}

When making a portfolio decision, according to Garlappi et al. (2007) and Lutgens and Schotman (2008), a robust investor may obtain $l$ priors $\pi^{i}(i=1, \cdots, l)$ from $l$ experts. However, the investor does not know which prior is the most reliable and hence combines all the priors to select a portfolio that may not necessarily be optimal for some experts' recommendations, but that may do a good job for the expert whose prior is true. To demonstrate how the proposed approach can be implemented in practice and to compare the portfolio performance from this model (RCVaR) to the nominal CVaR (Rockafellar and Uryasev, 2000) and WCVaR (Zhu and Fukushima, 2005), we consider in this section the robust portfolio optimization problem with multiple experts. In-sample and out-of-sample performances are compared.

\subsection{Equity portfolio management}

To investigate whether WCVaR works well in the case of a normal distribution, we consider in this subsection a portfolio using 30 industry portfolios as individual assets. The data are available from the website of Professor Kenneth French. ${ }^{6}$ Monthly returns are used from January 1997 to December 2006, i.e., 120 observations.

We split the sample period into four non-overlapped sub-samples (30 observations for each sub-sample) by assuming that there are four experts hired by the investor, and that each of them uses one sub-sample to obtain the prior of the portfolio return distribution. The Jarque-Bera test is used to determine if the portfolio return can be generally regarded as multivariate normal distribution. The test failed 24 times at the $1 \%$ level and 30 times at the $5 \%$ level for the 120 tests performed. ${ }^{7}$ This allows us to assume the returns are approximately normal as in Goldfarb and Iyengar (2003b) and Garlappi et al. (2007), and use $\left(P_{2}\right)$ to make the portfolio decision.

In this example, we set $\alpha=0.95, \bar{x}=\boldsymbol{e}$, and $\underline{x}=0$. To compare the performances of the nominal CVaR, WCVaR, and RCVaR optimal portfolios for various values of the required minimal expected/worst-case expected return $\mu$, Table 1 reports the expected values and the CVaRs at the 0.95 confidence level of the corresponding portfolios. It should be mentioned that the numerical experiments for the nominal CVaR and WCVaR are also performed via the second-order cone program $\left(P_{2}\right)$. For the WCVaR strategy, we simply remove $\gamma^{*}\left(\bar{y}^{i}, \Sigma^{i}\right)$ from $\left(P_{2}\right)$ and for the nominal CVaR strategy, we use the full sample for the mean-covariance forecasts by removing $\gamma^{*}\left(\bar{y}^{i}, \Sigma^{i}\right)$ and letting $l=1$ in $\left(P_{2}\right)$. Thus, we can compute the actual

\footnotetext{
${ }^{6}$ http://mba.tuck.dartmouth.edu/pages/faculty/ken.french/data-library.him.

${ }^{7}$ The basic statistics of the portfolio returns are available upon request.
} 
Table 1: Comparison of performances of CVaR, WCVaR, and RCVaR

\begin{tabular}{|c|c|c|c|c|c|c|c|c|c|}
\hline \multirow{2}{*}{$\mu$} & & \multicolumn{4}{|c|}{ Mean } & \multicolumn{4}{|c|}{$\mathrm{CVaR}_{0.95}$} \\
\hline & & expert1 & expert2 & expert3 & expert 4 & expert1 & expert2 & expert3 & expert 4 \\
\hline \multirow{3}{*}{1.15} & CVaR & 1.4201 & 1.1248 & 1.4368 & 1.2702 & 6.2231 & 4.0274 & 5.8595 & 2.9416 \\
\hline & WCVaR & 1.4329 & 1.1725 & $1.4612^{*}$ & 1.3318 & 6.0477 & 4.2376 & 6.0477 & 3.0104 \\
\hline & RCVaR & 1.4719 & 1.1500 & $1.5394^{* *}$ & 1.2696 & 6.3840 & 4.1813 & 5.6466 & 3.0071 \\
\hline \multirow{3}{*}{1.20} & CVaR & 1.4201 & 1.1248 & 1.4368 & 1.2702 & 6.2231 & 4.0274 & 5.8595 & 2.9416 \\
\hline & WCVaR & 1.4304 & 1.2000 & $1.4795^{*}$ & 1.3260 & 6.0491 & 4.2304 & 6.0491 & 3.0917 \\
\hline & RCVaR & 1.4656 & 1.2000 & $1.5790^{* *}$ & 1.2595 & 6.4025 & 4.2001 & 5.6412 & 3.1633 \\
\hline \multirow{3}{*}{1.25} & CVaR & 1.4201 & 1.1248 & 1.4368 & 1.2702 & 6.2231 & 4.0274 & 5.8595 & 2.9416 \\
\hline & WCVaR & 1.4258 & 1.2500 & $1.5128^{*}$ & 1.3157 & 6.0589 & 4.2429 & 6.0589 & 3.2527 \\
\hline & RCVaR & 1.4556 & 1.2500 & $1.6238^{* *}$ & 1.2513 & 6.4353 & 4.2597 & 5.6411 & 3.3317 \\
\hline \multirow{3}{*}{1.30} & CVaR & 1.4201 & 1.1248 & 1.4368 & 1.2702 & 6.2231 & 4.0274 & 5.8595 & 2.9416 \\
\hline & WCVaR & 1.4210 & 1.3000 & $1.5459^{*}$ & 1.3058 & 6.0780 & 4.2871 & 6.0780 & 3.4290 \\
\hline & RCVaR & 1.4655 & 1.3000 & $1.7115^{* *}$ & 1.3000 & 6.5203 & 4.5349 & 5.6905 & 3.5992 \\
\hline \multirow{3}{*}{1.35} & CVaR & 1.4589 & 1.1485 & 1.5085 & 1.2841 & 6.2867 & 4.1583 & 5.7522 & 2.9836 \\
\hline & WCVaR & 1.4175 & 1.3500 & $1.6263^{*}$ & 1.3500 & 6.1470 & 4.5536 & 6.1470 & 3.7245 \\
\hline & RCVaR & 1.4488 & 1.3500 & $1.7723^{* *}$ & 1.3500 & 6.6665 & 4.7593 & 5.7983 & 4.0038 \\
\hline \multirow{3}{*}{1.40} & CVaR & 1.4815 & 1.2227 & 1.6115 & 1.2843 & 6.3923 & 4.3239 & 5.6745 & 3.2209 \\
\hline & WCVaR & 1.4305 & 1.4000 & $1.6788^{*}$ & 1.4000 & 6.2701 & 4.8114 & 6.2701 & 4.2213 \\
\hline & RCVaR & 1.4000 & 1.4000 & $1.7907^{* *}$ & 1.4000 & 6.8784 & 4.8766 & 5.9678 & 4.1733 \\
\hline \multirow{3}{*}{1.45} & CVaR & 1.4831 & 1.3235 & 1.7265 & 1.2669 & 6.5915 & 4.5295 & 5.6428 & 3.5821 \\
\hline & WCVaR & 1.4500 & 1.4500 & $1.7270^{*}$ & 1.4500 & 6.4232 & 5.1073 & 6.4232 & 4.7753 \\
\hline & RCVaR & 1.4500 & 1.4500 & $1.8426^{* *}$ & 1.4500 & 7.2272 & 5.0345 & 6.2690 & 4.4799 \\
\hline \multirow{3}{*}{1.50} & CVaR & 1.4760 & 1.4235 & 1.8355 & 1.2650 & 6.8556 & 4.7871 & 5.7302 & 3.9338 \\
\hline & WCVaR & 1.5000 & 1.5000 & $1.7590^{*}$ & 1.5000 & 6.6127 & 5.4344 & 6.6127 & 5.4418 \\
\hline & RCVaR & 1.5000 & 1.5000 & $1.9108^{* *}$ & 1.5000 & 7.6484 & 5.3408 & 6.6374 & 4.8704 \\
\hline \multirow{3}{*}{1.55} & CVaR & 1.4827 & 1.4987 & 1.9266 & 1.2920 & 7.1476 & 5.0874 & 5.8521 & 4.3872 \\
\hline & WCVaR & 1.5500 & 1.5500 & $1.8140^{*}$ & 1.5500 & 6.9218 & 5.9025 & 6.9218 & 6.2588 \\
\hline & RCVaR & 1.5500 & 1.5500 & $1.9764^{* *}$ & 1.5500 & 8.1233 & 5.7432 & 7.0548 & 5.3260 \\
\hline
\end{tabular}

Note: ${ }^{*}$ and ${ }^{* *}$ represent the best returns of the WCVaR and RCVaR optimal portfolios, respectively. 
expected returns and the corresponding CVaRs when the investor adopts one particular expert's advice.

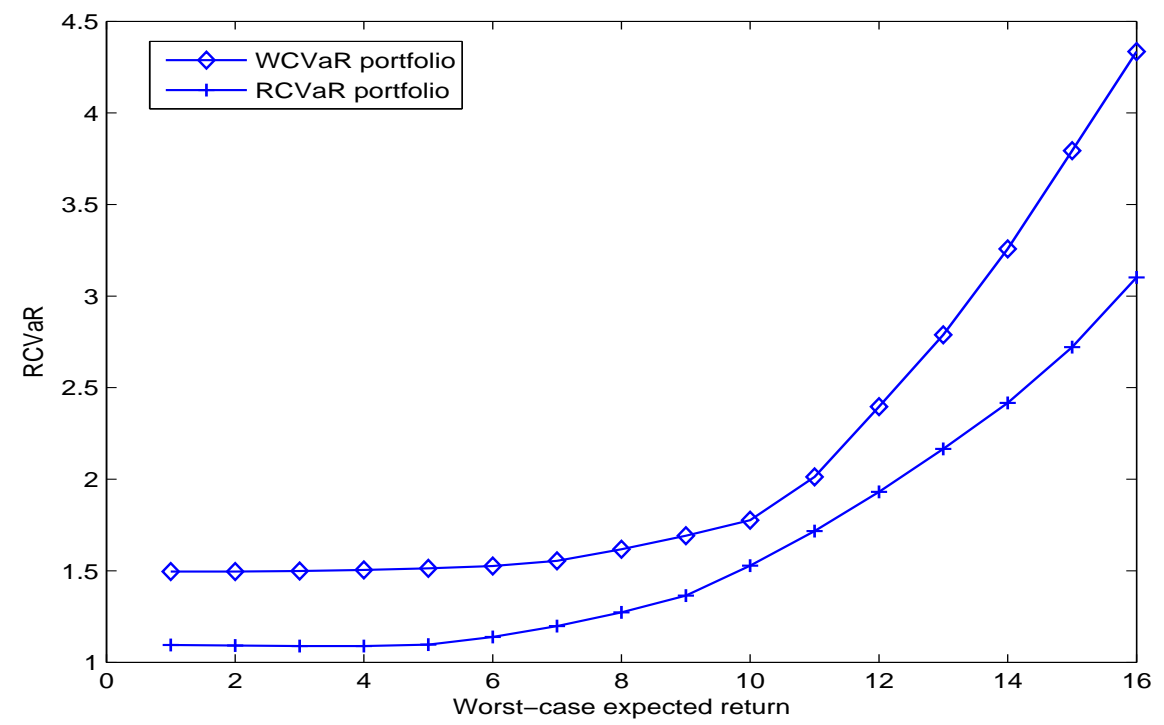

Figure 1: Relative robust CVaRs of the WCVaR and RCVaR optimal portfolios

From Table 1, it is clear that the larger the required minimal expected/worst-case expected returns, the larger the associated risk. For the same value of $\mu$, the risk of the WCVaR optimal portfolios appears to be larger than that of the nominal optimal portfolio strategy, while the risk of the RCVaR's is larger than that of the CVaR's. For example, when $\mu=1.35$, the risk of the CVaR optimal portfolio is 6.2867 , which is larger than the WCVaR risk (6.1470), but less than 6.6665, the risk of the RCVaR optimal portfolio. However, higher risk is compensated for by higher return. In fact, the larger values of the RCVaRs do not necessarily imply higher risk than that of the nominal CVaR and the WCVaR policies, which is only because the investor considers more uncertainty of future extreme scenarios and hence takes a conservative strategy. For both the WCVaR and RCVaR formulations, the worst-case expected return can be guaranteed no matter which expert's forecast is correct.

On the other hand, we can also find from Table 1 that for each worst-case expected return $\mu$, while the worst-case return is ensured, the best-case return of RCVaR is higher than that of WCVaR. For example, when $\mu=1.40$, the best-case return of the WCVaR optimal portfolio is 1.6788 , while the RCVaR optimal portfolio best-case return is $1.7907 .{ }^{8}$ This means that the RCVaR strategy considers not only the worst-case scenario but also the best-case scenario. Finally, we show the worst-case return versus the relative CVaR risk efficient frontier in Figure 1. In the computation of the RCVaR risk for the WCVaR optimal

\footnotetext{
${ }^{8}$ Notice here that a monthly mean return of $1.7907 \%$ is not unbelievable since for the 1997 to 2006 period, most of the industry index returns in our data had a monthly mean return in excess of $1.2 \%$ with the largest being $2.4 \%$
} 
portfolio $x^{*}$, we replace $x$ in $\left(P_{2}\right)$ by $x^{*}$ and solve the problem. As expected, the RCVaR strategy will do a better job than the WCVaR strategy when the investor faces multiple priors and does not know which one is most reliable; this is very important for the active portfolio investor who may make a robust but not necessarily too conservative decision.

\subsection{Hedge fund portfolio management}

In this subsection, 13 Credit Suisse/Tremont Hedge Fund Indices (listed in Table 2) are selected as the candidates for constructing hedge fund portfolios, the returns of which are asymmetrically distributed.

Table 2: Credit Suisse/Tremont Hedge Fund Indices

\begin{tabular}{c|l}
\hline \hline 1 & Convertible Arbitrage \\
2 & Dedicated Short Bias \\
3 & Emerging Markets \\
4 & Equity Market Neutral \\
5 & Event Driven \\
6 & Distressed \\
7 & Multi-Strategy \\
8 & Risk Arbitrage \\
9 & Fixed Income Arbitrage \\
10 & Global Macro \\
11 & Long/Short Equity \\
12 & Managed Futures \\
13 & Multi-Strategy \\
\hline \hline
\end{tabular}

Monthly values and returns of those indices, from April 1994 to December 2008, can be freely downloaded from http://www.hedgeindex.com. The returns differently behave during different time periods (Figure 2). In 1994, the returns exhibited lower expected values than those in time periods 1995 2000 and 2001 2005. Furthermore, the returns within 1995 2000 are more volatile than those within 2001 2005. Suppose the returns within these three time periods are the possible multiple distributions that are chosen by three experts to construct robust portfolios. The data after 2005 are used to test out-of-sample performance.

Notice that the returns of hedge fund indices are asymmetrical. We employ model $\left(P_{1}\right)$ to optimize the portfolios. However, there are in total only 178 monthly returns, which is very limited as the input samples for the model $\left(P_{1}\right)$. To generate stable solutions, we have to generate more samples according to each possible distribution. Here, we generate sufficient samples by discretizing the principal component of the returns (for a discussion of this method, see Topaloglou, Vladimirou and Zenios, 2002). 


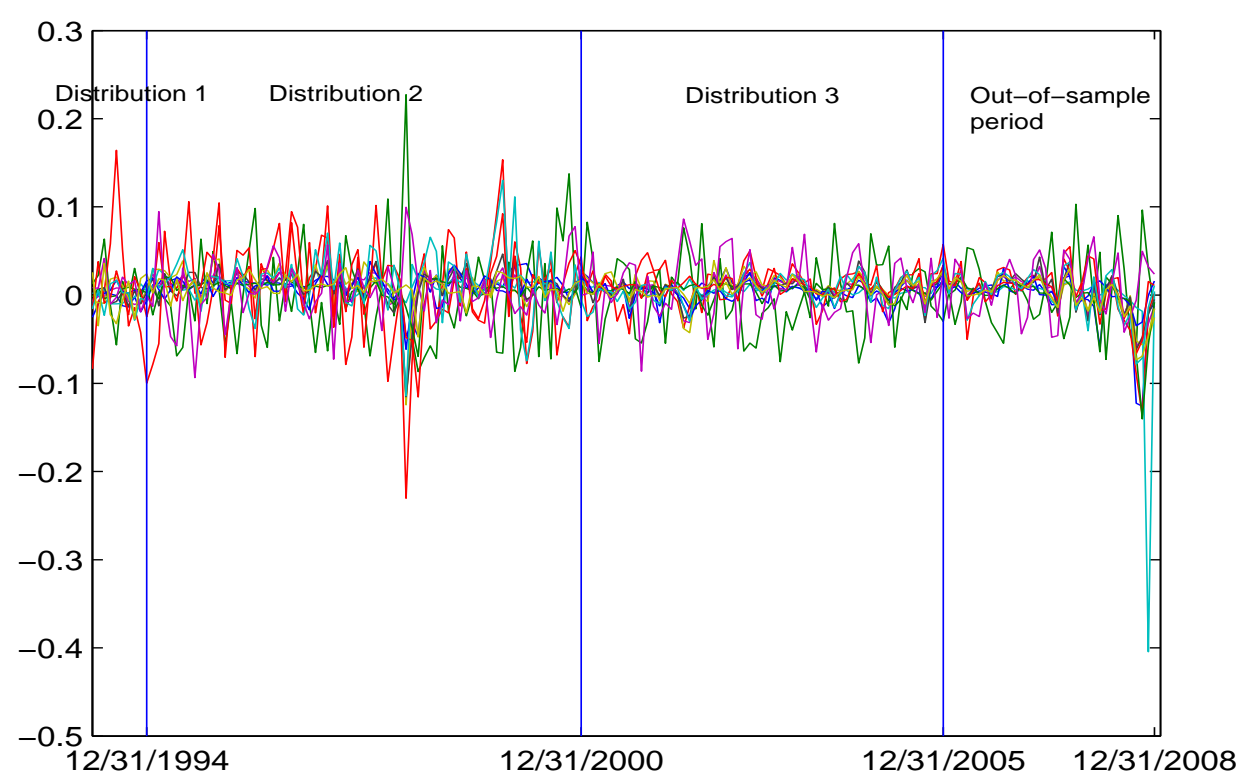

Figure 2: Returns of Credit Suisse/Tremont Hedge Fund Indices (Apr. $1994 \sim$ Dec. 2008)

To compare the performance of the CVaR model, WCVaR model, and RCVaR model, we generate four portfolios with each model by setting different minimum expected return $\mu$ 's, which almost span the mean-CVaR (WCVaR, RCVaR) efficient frontier. We set $\alpha=0.95$, $\bar{x}=e$, and $\underline{x}=0$. For the CVaR model, these three possible distributions are integrated as one nominal distribution. The out-of-sample evolution of portfolio values according to these efficient portfolios are illustrated in Figures 3 and 4, where the initial wealth is set at 1 . Figure 3 covers the time period from January 2006 to December 2008, during which the Tremont Hedge Fund Index increased in 2006 and 2007 and then decreased in 2008. Figure 4 covers the time period from January to December 2008, during which we witnessed a bear market (i.e., the Tremont Hedge Fund Index decreased). The robust portfolios may lose potential opportunities in a bull market, however, they perform much better in a bear market as compared to the portfolios that do not consider the uncertainty of the distribution. Robustness with respect to distribution uncertainty is especially necessary from the view point of downside risk reduction. For this hedge fund example, however, different from the previous equity portfolio management problem, the portfolios generated by the WCVaR and RCVaR models do not exhibit a notable difference. 


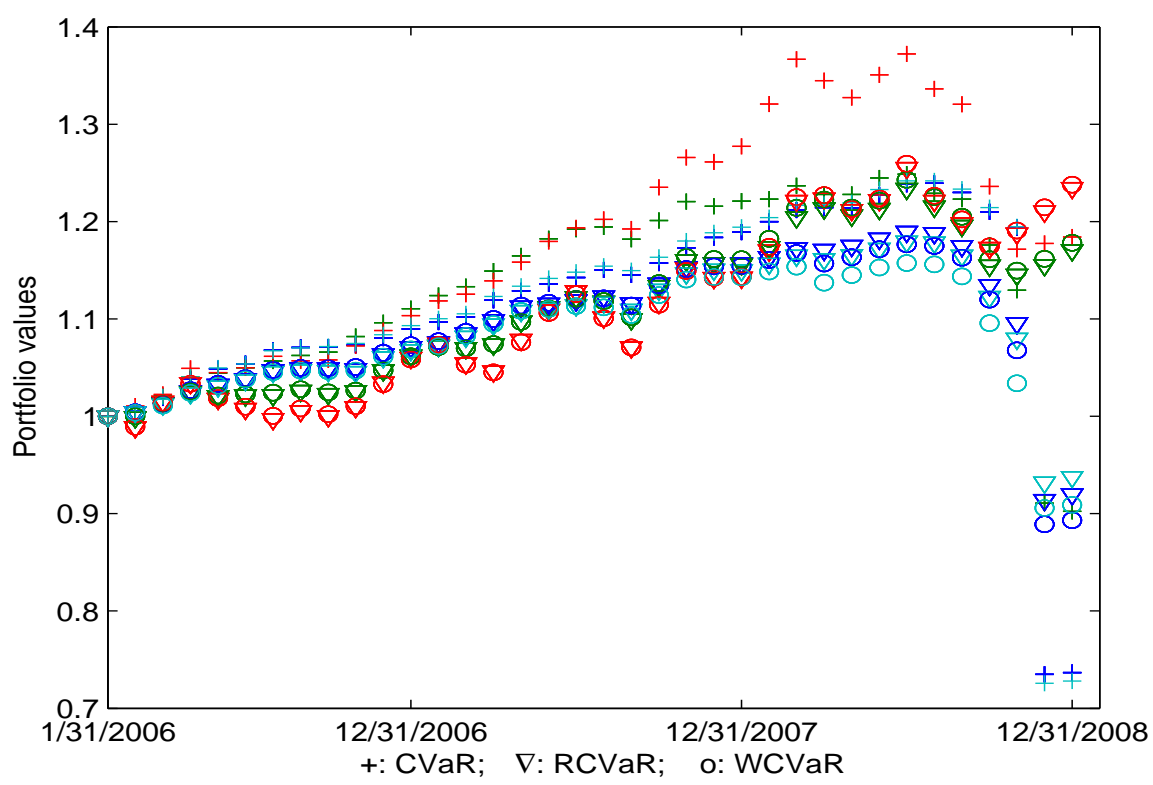

Figure 3: Out-of-sample evolution of values of portfolios (Jan. $2006 \sim$ Dec. 2008)

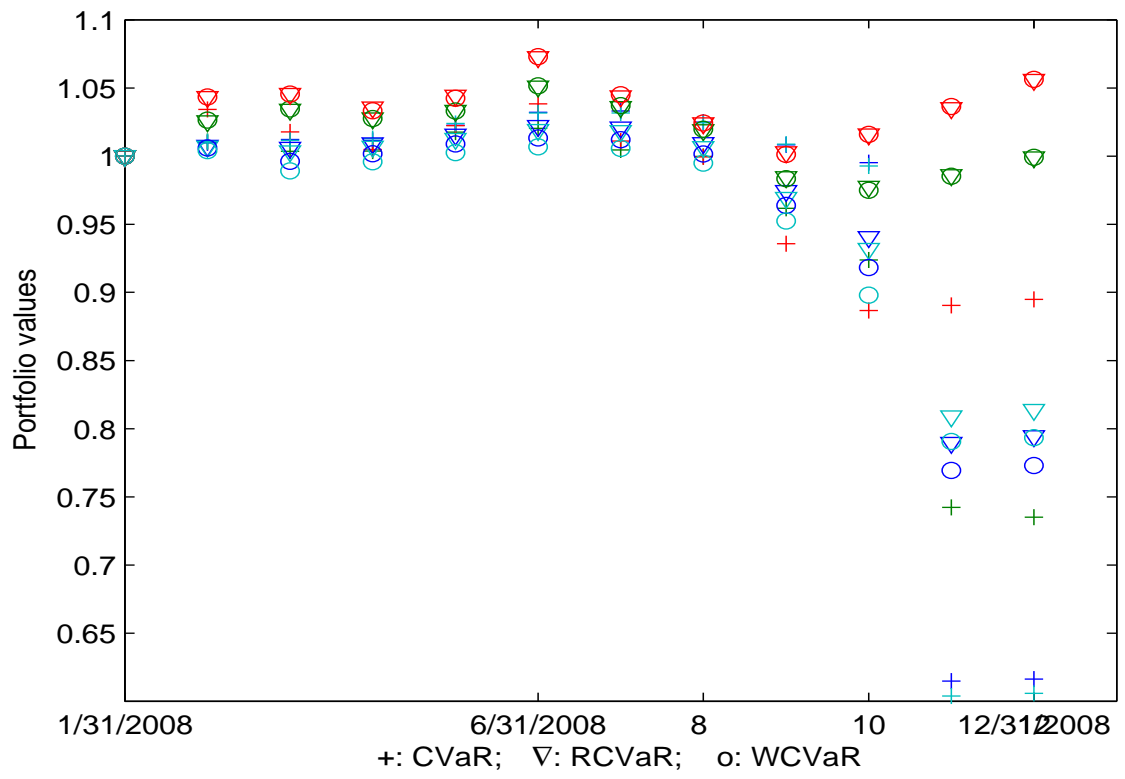

Figure 4: Out-of-sample evolution of values of portfolios (Jan. $2008 \sim$ Dec. 2008) 


\section{Conclusion}

In this paper, we propose a relative robust CVaR model for an investor who can make a robust but not very conservative portfolio decision. In contrast to the worst-case CVaR approach developed by Zhu and Fukushima (2005), our approach takes into account the best-case situation of the input data in addition to the worst-case scenario. We illustrate how to use the proposed model in the robust portfolio optimization problem with multiple experts.

\section{References}

[1] Artzner, P., F. Delbaen, J.-M. Eber and D. Heath (1999) Coherent Measures of Risk, Mathematical Finance, 9, 203-228.

[2] Ben-Tal, A. and A. Nemirovski (1998) Robust Convex Optimization, Mathematics of Operations Research, 23, 769-805.

[3] Ben-Tal, A. and A. Nemirovski (1999) Robust Solutions of Uncertain Linear Programs, Operations Research Letters, 25, 1-13.

[4] Best, M.J. and R.R. Grauer (1991) On the Sensitivity of Mean-Variance Efficient Portfolios to Changes in Asset Means: Some Analytical and Computational Results, Review of Financial Studies, 4(2), 315-342

[5] Black, F., and R. Litterman (1992) Global Portfolio Optimization, Financial Analysts Journal, 48, 28-43.

[6] Broadie, M. (1993) Computing Efficient Frontiers using Estimated Parameters, Annals of Operations Research: Special Issue on Financial Engineering, 45, 21-58.

[7] Chopra, V.K. (1993) Mean-Variance Revisited: Near-Optimal Portfolios and Sensitivity to Input Variations, Journal of Investing, 2(1), 51-59.

[8] Chopra, V.K. and W.T. Ziemba (1993) The Effects of Errors in Means, Variances, and Covariances on Optimal Portfolio Choice, Journal of Portfolio Management, 19(2), 6-11.

[9] Costa, O.L. and A.C. Paiva (2002) Robust Portfolio Selection Using Linear-Matrix Inequality, Journal of Economic Dynamics \& Control, 26, 889-909.

[10] El Ghaoui, L. and H. Lebret (1997) Robust Solutions to Least-Squares Problems with Uncertain Data, SIAM Journal on Matrix Analysis and Applications, 18, 1035-1064.

[11] El Ghaoui, L., M. Oks, and F. Oustry (2003) Worst-Case Value-at-Risk and Robust Portfolio Optimization: A Conic Programming Approach, Operations Research, 51, 543556.

[12] Fabozzi, F.J., P.N. Kolm, D. Pachamanova, and S.M. Focardi (2007) Robust Portfolio Optimization and Management, John Wiley \& Sons, Hoboken, NJ. 
[13] Fabozzi, F.J., D. Huang, and G. Zhou (2009) Robust Portfolios: Contributions from Operations Research and Finance, to appear in Annals of Operations Research.

[14] Garlappi, L., R. Uppal, and T. Wang (2007) Portfolio Selection with Parameter and Model Uncertainty: A Multi-Prior Approach, Review of Financial Studies, 20(1) 41-81.

[15] Goldfarb, D. and G. Iyengar (2003a) Robust Convex Quadratically Constrained Programs, Mathematical Programming, 97, 495-515.

[16] Goldfarb, D. and G. Iyengar (2003b) Robust Portfolio Selection Problems, Mathematics of Operations Research, 28, 1-38.

[17] Gülpinar and B. Rustem (2007) Worst-Case Robust Decisions for Multi-Period MeanVariance Portfolio Optimization, European Journal of Operational Research, 193(3), 981-1000.

[18] Huang, D., S.S. Zhu, F.J. Fabozzi, and M. Fukushima (2008) Portfolio Selection with Uncertain Exit Time: A Robust CVaR Approach, Journal of Economic Dynamics and Control, 32(2), 594-623.

[19] Huang, D., F.J. Fabozzi, and M. Fukushima (2007) Robust Portfolio Selection with Uncertain Exit Time Using Worst-Case VaR Strategy, Operations Research Letters, 627635 .

[20] Knight, F. (1921) Risk, Uncertainty and Profit, Houghton Mifflin, Boston, MA.

[21] Krokhmal, P., R. Murphey, P. Pardalos and S. Uryasev (2004) Use of Conditional Valueat-Risk in Stochastic Programs with Poorly Defined Distributions, S. Butenko et al (Eds.) Recent Developments in Cooperative Control and Optimization, Kluwer Academic Publishers, 225-243.

[22] Krokhmal, P., R. Murphey, P. Pardalos, S. Uryasev and G. Zrazhevsky (2003) Robust Decision Making: Addressing Uncertainties in Distributions. S. Butenko et al (Eds.) Cooperative Control: Models Applications and Algorithms. Kluwer Academic Publishers, 165-185.

[23] Liesiö, J., P. Mild and A. Salo (2007) Preference Programming for Robust Portfolio Modeling and Project Selection, European Journal of Operational Research, 181(3), 1488-1505.

[24] Litterman, R. (2003) Modern Investment Management: An Equilibrium Approch, John Wiley \& Sons, Hoboken, NJ.

[25] Lu, Z. (2006) A New Cone Programming Approach for Robust Portfolio Selection. Working paper, http://www.math.sfu.ca/ zhaosong/ResearchPapers/robport.pdf.

[26] Lutgens F. and P. Schotman (2008) Robust Portfolio Optimization with Multiple Experts, to appear in Review of Finance.

[27] Markowitz, H.M. (1952) Portfolio Selection, Journal of Finance, 7, 77-91. 
[28] Natarajan, K., D. Pachamanova, M. Sim (2005) Constructing Risk Measures from Uncertainty Sets, to appear in Operations Research.

[29] Natarajan, K., D. Pachamanova and M. Sim (2008) Incorporating Asymmetric Distributional Information in Robust Value-at-Risk Optimization, Management Science, 54, 573-585.

[30] Rockafellar, R.T. and S. Uryasev (2000) Optimization of Conditional Value-at-Risk, Journal of Risk, 2, 21-41.

[31] Rockafellar R.T. and S. Uryasev (2002) Conditional Value-at-Risk for General Loss Distributions, Journal of Banking and Finance, 26(7), 1443-1471.

[32] Rockafellar, R. T., S. Uryasev and M. Zabarankin (2006) Generalized Deviations in Risk Analysis, Finance and Stochastics, 10, 51-74.

[33] Shen, R. and S. Zhang (2008) Robust Portfolio Selection Based on a Multi-Stage Scenario Tree, European Journal of Operational Research, 191(3), 864-887.

[34] Stinstra, E. and D.D. Hertog (2008) Robust Optimization Using Computer Experiments, European Journal of Operational Research, 191(3), 816-837.

[35] Topaloglou, N., H. Vladimirou, S. A. Zenios (2002) CVaR Models with Selective Hedging for International Asset Allocation. Journal of Banking and Finance 26, 1535-1561.

[36] Tütüncü, R. and M. Koenig (2004) Robust Asset Allocation, Annals of Operations Research, 132, 157-187.

[37] Yamai, Y. and T. Yoshiba (2005) Value-at-Risk versus Expected Shortfall: A Practical Perspective, Journal of Banking \& Finance, 29, 997-1015.

[38] Zhu, S. S. and M. Fukushima (2005) Worst-Case Conditional Value-at-Risk with Application to Robust Portfolio Management, to appear in Operations Research.

[39] Zhu, S. S., D. Li and S. Y. Wang (2007) Robust Portfolio Selection under Downside Risk Measures. Working paper, http://www.fdms.fudan.edu.cn/teacherhome /sszhu/paper/ZLW_WLPM_QF.pdf. 